\title{
AVALIAÇÃO DA BIODEGRADAÇÃO DE MATRIZES POROSAS À BASE DE HIDROXIAPATITA PARA APLICAÇÃO COMO FONTES RADIOATIVAS EM BRAQUITERAPIA
}

\author{
Kássio André Lacerda* e Fernando Soares Lameiras \\ Centro de Desenvolvimento da Tecnologia Nuclear, Comissão Nacional de Energia Nuclear, 31270-901 Belo Horizonte - MG, \\ Brasil \\ Viviane Viana Silva \\ Universidade Vale do Rio Verde de Três Corações, 37410-000 Três Corações - MG, Brasil
}

Recebido em 26/6/08; aceito em 14/1/09; publicado na web em 28/5/09

\begin{abstract}
EVALUATION OF THE BIODEGRADATION OF POROUS HYDROXYAPATITE MATRICES FOR APPLICATION AS RADIOACTIVE SOURCES IN BRACHYTHERAPY. Porous ceramic materials based on calcium phosphate compounds (CPC) have been studied aiming at different biomedical applications such as implants, drug delivery systems and radioactive sources for brachytherapy. Two kinds of hydroxyapatite (HAp) powders and their ceramic bodies were characterized by a combination of different techniques (XRF, BET method, SEM, ICP/AES and neutron activation analysis - NAA) to evaluate their physico-chemical and microstructural characteristics in terms of chemical composition, segregated phases, microstructure, porosity, chemical and thermal stability, biodegradation and incorporation of substances in their structures. The results revealed that these systems presented potential for use as porous biodegradable radioactive sources able to be loaded with a wide range of radionuclides for cancer treatment by the brachytherapy technique.
\end{abstract}

Keywords: hydroxyapatite; biodegradation; brachytherapy.

\section{INTRODUÇÃO}

Os biomateriais surgem dos avanços científicos e tecnológicos impulsionados pela demanda por novos materiais. Um dos segmentos em ascendência na área de biomateriais é o desenvolvimento de estruturas cerâmicas à base de fosfato de cálcio, tendo em vista a sua biocompatibilidade, características físico-químicas e microestruturais e propriedades mecânicas similares aos dos tecidos duros. ${ }^{1,2}$ Matrizes sintéticas de hidroxiapatita de cálcio (HAp), principal fosfato de cálcio constituinte dos tecidos ósseos e dentários, vêm sendo alvo de investigações, tendo em vista sua potencialidade para aplicações como material para implantes, ${ }^{3-5}$ sistemas para liberação de drogas, ${ }^{6}$ fontes radioativas para braquiterapia e engenharia de tecidos. ${ }^{7-12}$

A HAp é um biocerâmico, de fórmula química $\mathrm{Ca}_{10}\left(\mathrm{PO}_{4}\right)_{6}(\mathrm{OH})_{2}$. Trata-se de um duplo sal de fosfato tricálcio e do hidróxido de cálcio, conforme Equação 1.

$3 \mathrm{Ca}_{3}\left(\mathrm{PO}_{4}\right)_{2}+\mathrm{Ca}(\mathrm{OH})_{2} \rightarrow \mathrm{Ca}_{10}\left(\mathrm{PO}_{4}\right)_{6}(\mathrm{OH})_{2}$

A Figura 1 apresenta a estrutura cristalina da hidroxiapatita no modelo de esferas rígidas. $\mathrm{O}$ arranjo atômico consiste de uma célula hexagonal, na qual os íons hidroxilas (círculo) estão localizados nos cantos da célula, rodeados por dois grupos de átomos de $\mathrm{Ca}_{\mathrm{II}}$ (triângulo-lado direito) e por dois grupos de $\mathrm{PO}_{4}{ }^{3-}$ tetraédricos (triângulo-lado esquerdo, o fósforo não está visível), ambos arranjados em posições triangulares. Os átomos de $\mathrm{Ca}_{\mathrm{I}}$ (hexágono) estão mais afastados segundo um arranjo hexagonal. A existência de dois sítios de íons cálcio traz consequências importantes para as hidroxiapatitas que contêm impurezas catiônicas, pois suas propriedades estruturais podem ser modificadas dependendo do sítio ocupado pelo cátion da impureza.

\footnotetext{
*e-mail:kal@cdtn.br
}

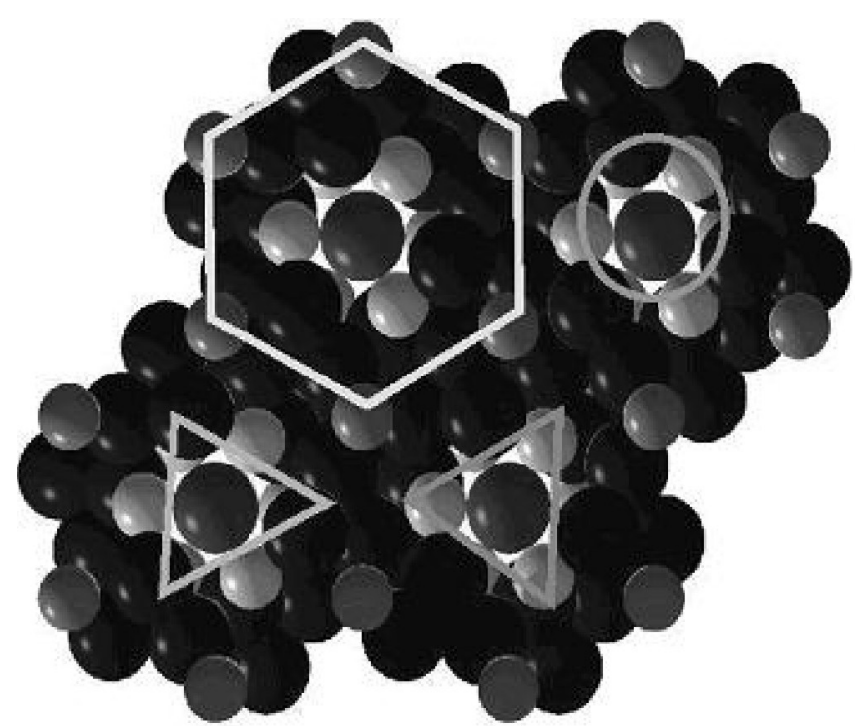

Figura 1. Estrutura cristalina da hidroxiapatita

O código de prática para física médica e procedimento clínico da braquiterapia trata do uso de pequenas fontes radioativas para tratamento in situ de câncer em diferentes locais do corpo. ${ }^{8}$ As vantagens da braquiterapia, quando comparada à terapia de feixe externo (teleterapia), são várias. ${ }^{10,11,13} \mathrm{~A}$ localização da dose no volume tumoral, os gradientes de dose em torno do implante e a homogeneidade da dose dentro do tumor são melhores que aqueles da teleterapia. ${ }^{10} \mathrm{Na}$ atualidade, as fontes de braquiterapia são compostas de um material onde são incorporados os radionuclídeos, marcadores radiopacos, e um encapsulamento metálico biocompatível. Estas fontes metálicas vêm apresentando alguns problemas e limitações, como atenuação da radiação, a não homogeneidade da dose e, em alguns casos, a necessidade de remoção das fontes do paciente depois do tratamento. ${ }^{13}$ 
Uma aplicação interessante para matrizes porosas biodegradáveis de HAp está direcionada à incorporação de diferentes radionuclídeos em suas estruturas para tratamento terapêutico de câncer pela técnica de braquiterapia, e o fato desta fonte ser bioabsorvível, torna possível a eliminação da fonte após o decaimento do radionuclídio incorporado.

O processo de biodegradação das HAp tem sido estudado, ${ }^{6,15-18}$ mostrando uma dependência de vários fatores, como composição química (associada à razão molar $\mathrm{Ca} / \mathrm{P})$, solubilidade e microestrutura do material, bem como $\mathrm{pH}$ no meio fisiológico e fatores biológicos.

Este trabalho consiste na avaliação da biodegradação das fontes de braquiterapia à base HAp em alguns tipos de fluidos e a determinação de seu tempo de utilização. Neste sentido, se faz necessário o conhecimento das características química, físico-química e microestrutura do material e sua capacidade de armazenamento de radionuclídio em sua estrutura, ${ }^{10,11,13}$ bem como do $\mathrm{pH}$ no meio fisiológico e fatores biológicos.

\section{PARTE EXPERIMENTAL}

\section{Matrizes de Hap}

Matrizes porosas de HAp foram preparadas utilizando pós sintético (HA) e comercial (HACL). ${ }^{13,14} \mathrm{O}$ pó sintético foi obtido através de rota coprecipitativa usando volumes iguais de soluções aquosas de $\mathrm{Ca}\left(\mathrm{NO}_{3}\right)_{2}$. $4 \mathrm{H}_{2} \mathrm{O} 1,0 \mathrm{~mol} / \mathrm{Le}\left(\mathrm{NH}_{4}\right)_{2} \mathrm{HPO}_{4}$ p.a. $0,6 \mathrm{~mol} / \mathrm{L}$. A síntese ocorreu em meio amoniacal, à temperatura ambiente e sob agitação. A solução permaneceu em descanso para a decantação do precipitado (Equação 2). Após o precipitado ter decantado, o mesmo foi filtrado e lavado sob vácuo.

$10 \mathrm{Ca}\left(\mathrm{NO}_{3}\right)_{2}+6\left(\mathrm{NH}_{4}\right)_{2} \mathrm{HPO}_{4}+8 \mathrm{NH}_{4} \mathrm{OH} \rightarrow \mathrm{Ca}_{10}\left(\mathrm{PO}_{4}\right)(\mathrm{OH})_{2} \downarrow+$ $6 \mathrm{H}_{2} \mathrm{O}+20 \mathrm{NH}_{4} \mathrm{NO}_{3} \uparrow$

A prensagem dos pós de HAp foi realizada em uma prensa hidráulica com matriz flutuante para simular a dupla ação. ${ }^{19}$ As prensagens foram realizadas sob uma pressão de $100 \mathrm{MPa},{ }^{14} \mathrm{o}$ diâmetro da matriz é de $0,81 \mathrm{~cm}$. A sinterização das matrizes de HAp foi realizada ao ar em um forno tipo mufla, marca Lavoisier, modelo $402 \mathrm{D}$, com controladora, a uma temperatura de $900^{\circ} \mathrm{C}$, por um período de $1 \mathrm{~h}$, com taxas de aquecimento/resfriamento de $10^{\circ} \mathrm{C} / \mathrm{min}$. Para os propósitos deste trabalho, a presença de porosidade aberta é importante para a incorporação de radionuclídeos. Assim, é importante que a sinterização das matrizes prossiga até o fim do primeiro estágio ou o início do segundo estágio, onde há um compromisso entre a boa resistência mecânica e a alta porosidade aberta. A composição química dos pós foi determinada utilizando-se um espectrômetro de fluorescência de raios X da Rigaku, modelo 3134, com tubo de cromo e cristais analisadores de fluoreto de lítio (LiF) e ditartarato de etileno de amina (EDDT).

Os valores de área superficial específica para os pós foram determinados a partir de um analisador BET, modelo Quantachrone Nova-1200, por adsorção de nitrogênio.

As características microestruturais dos corpos cerâmicos (forma e tamanho de partículas, poros, grãos, aglomerados) foram avaliadas, utilizando-se um microscópio eletrônico de varredura Jeol, modelo JSM-840A, do Laboratório de Microscopia Eletrônica e Microanálise, no Departamento de Física/UFMG do consórcio UFMG-CDTN.

As medidas de densidade e de porosidade (aberta e total) dos corpos cerâmicos obtidos foram determinadas pelos métodos de penetração e imersão em xilol e geométrico.

\section{Impregnação das matrizes}

O processo de impregnação foi realizado por imersão das matrizes cerâmicas em uma solução de $\mathrm{NaI}$ (iodeto de sódio), variando a con- centração dessa solução de 0,5 a 1,0 mol/L,,$^{13,20,21}$ e com $\mathrm{pH}$ corrigido para 12 com $\mathrm{NaOH} .^{22,23}$

\section{Caracterização das matrizes impregnadas}

Para ser aplicada, a técnica de AAN é necessário que se disponha de um irradiador de nêutrons. Utilizou-se o reator de pesquisa Triga Mark I IPR-R1 do CDTN/CNEN. As matrizes impregnadas e padrões foram irradiados na mesa giratória do reator. O procedimento para esta análise, usual no caso da técnica ser aplicada de forma instrumental e ser comparativa, consistiu, inicialmente, em se acondicionar as amostras em seus respectivos tubos de polietileno. Uma vez lacrados, eles foram inseridos em outros tubos, denominados "coelhos", adequados para a inserção dos tubos de polietileno na mesa giratória do reator. Neste local, a uma potência de $100 \mathrm{~kW}$, o fluxo de nêutrons térmicos é de $6,6 \times 10^{11}$ nêutrons $\mathrm{cm}^{-2} \mathrm{~s}^{-1}$. A cada irradiação, as amostras foram acompanhadas por padrões do elemento de interesse, no caso, iodo. As amostras e padrões foram irradiados por $5 \mathrm{~min}$. Durante a irradiação ocorre a seguinte reação (Equação 3):

${ }^{127} \mathrm{I}+$ nêutron $\rightarrow{ }^{128} \mathrm{I}+$ gama + beta

Após a irradiação esperou-se um tempo adequado para o decaimento dos radionuclídeos de meias vidas mais curtas, tais como o $\mathrm{Na}$ (sódio), que poderiam interferir na espectrometria gama. Como o $\mathrm{Na}$ apresentava uma baixa concentração, não houve interferência.

Foi utilizada a técnica de espectrometria gama para a contagem das amostras após um tempo de decaimento de $2 \mathrm{~h}$. Cada amostra foi contada por um tempo de $200 \mathrm{~s}$ para que fosse alcançada uma boa estatística de contagem. A meia vida do ${ }^{128}$ I é de $24 \mathrm{~min}$.

O sistema de detecção gama consiste de detector HPGe, modelo GC5019, eficiência nominal de 50\%, marca Canberra, resolução de $1,9 \mathrm{keV}$ para a energia de $1332 \mathrm{keV}$ do ${ }^{60} \mathrm{Co}$ e eletrônica associada, interligado a microcomputador, com o programa de análise de espectros, Gennie 2000 Canberra. A geometria de contagem foi mantida constante para cada irradiação. De cada espectro levantado, foi obtida a área sob o pico de energia gama de $442 \mathrm{keV}$, característico do ${ }^{128}$ I. Para o cálculo da concentração, foi corrigido o tempo de decaimento do ${ }^{128}$ I para cada amostra e padrão. Com os dados dos padrões - área e concentração - foi construída a reta que descreve o comportamento da área em função da concentração. Nesta reta foram interpoladas as áreas do pico gama do iodo obtidas para as amostras, calculando-se as concentrações presentes. As concentrações também foram obtidas usando-se a equação da reta. ${ }^{24}$ Como foi uma análise comparativa, foram utilizados padrões do elemento em estudo, o iodo. ${ }^{13}$

\section{Testes de biodegradação}

Amostras (300 mg) de matrizes cerâmicas HA e HACL foram imersas, separadamente, em $50 \mathrm{~mL}$ de solução de soro fisiológico (solução aquosa $0,9 \%$ em $\mathrm{NaCl}$ ) sob agitação constante e à temperatura de $37,0 \pm 1,0{ }^{\circ} \mathrm{C}$. Os experimentos foram realizados em diferentes tempos de imersão: 0, 15, 30 e 45 min, 1, 3, 6 e 24 h. Alíquotas de $5 \mathrm{~mL}$ foram retiradas ao final de cada tempo para determinação da concentração de cálcio e fósforo (em termos de fosfato), e repostas com soro fresco. Os resultados foram analisados como porcentagem cumulativa de cálcio ou fosfato liberado no meio em função do tempo. Espectrometria de emissão atômica por plasma indutivamente acoplado (ICP/AES) foi utilizada para determinação da concentração de cálcio e fósforo liberados na solução fisiológica após imersão das amostras, a partir de um espectrômetro Espectroflame da Analytical Instruments. 


\section{RESULTADOS E DISCUSSÃO}

Na Tabela 1 estão representados os dados de composição química, área superficial, densidade e porosidade para os pós e corpos cerâmicos investigados. A composição química do pó de HA apresentou teores de cálcio e fósforo superiores aos do pó de HACL. A razão molar $\mathrm{Ca} / \mathrm{P}$ é um parâmetro para se avaliar o comportamento biodegradável de um material em fluidos fisiológicos. A degradação é função da estequiometria; quando a razão molar $\mathrm{Ca} / \mathrm{P}$ for diferente do valor estequiométrico de $1,67,{ }^{17}$ o material degrada e é absorvido pelo corpo. O pó de $\mathrm{HA}$ apresentou um valor de razão molar $\mathrm{Ca} / \mathrm{P}$ próximo ao estequiométrico. Por sua vez, o pó de HACL revelou um valor abaixo do valor estequiométrico $(1,67)$, exibindo características típicas de hidroxiapatita carbonatada. Os valores de áreas superficiais específicas demonstrados na Tabela 1 para ambos os pós estão próximos àqueles reportados na literatura para hidroxiapatita. ${ }^{13}$

Os métodos de penetração e imersão em xilol (PI) e geométrico (G) foram empregados na determinação da densidade, porosidade aberta e total dos corpos cerâmicos. Os valores de densidade obtidos por ambos métodos e amostras foram próximos e se apresentaram na ordem de $1,4 \mathrm{~g} / \mathrm{m}^{3}$. Os dois pós estudados revelaram uma baixa densificação do material com porosidade total da ordem de $55 \%$ e porosidade aberta da ordem de $54 \%$.

Os resultados de difração de raios X (Figura 2) revelaram que ambos os pós são cristalinos, com predominância de hidroxiapatita em temperaturas abaixo de $1000{ }^{\circ} \mathrm{C}$, embora exista a presença de fases amorfas. ${ }^{14}$ Verificou-se que o pó HACL é constituído, basicamente, de hidroxiapatita de cálcio carbonatada, enquanto que o pó de HA apresenta

(a)

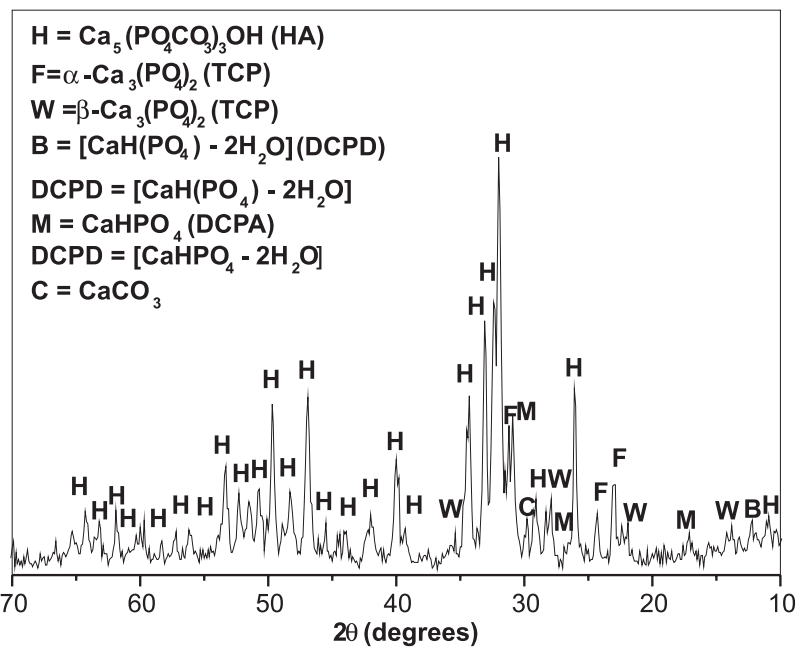

hidroxiapatita de cálcio como fase predominante. A presença de fases minoritárias solúveis, como fosfato de tricálcio ( $\alpha$ e $\beta$-TCP), fosfato de dicálcio hidratado (DCPD) e anidro (DCPA), $\mathrm{e} \mathrm{CaCO}_{3}{ }^{13,25}$ foi constatada nos difratogramas de raios $\mathrm{X}$ de ambos os pós. A ocorrência dessas fases solúveis sugere um possível comportamento biodegradável em fluidos fisiológicos.

As micrografias referentes aos corpos cerâmicos (matrizes) de HA e HACL estão apresentadas nas Figuras 3 e 4, respectivamente. Imagens do topo das matrizes podem ser visualizadas com aumentos de 10.000 e 20.000 vezes. A fase mais escura representa a fase hidroxiapatita, e a clara são pequenos grãos de HAp aderidos por atração física na superfície da matriz. As morfologias de ambas as matrizes são similares, ${ }^{4,13}$ com formação predominante de grãos de maiores dimensões e contornos bem definidos. ${ }^{19}$ Entretanto, a microestrutura relativa à matriz de HACL (Figura 4) revelou presença de grãos de maior dimensão em relação à matriz de HA (Figura 3), tendo em vista a granulometria mais fina e maior área superficial do pó comercial (Tabela 1). A alta porosidade exibida por ambas as matrizes pode ser atribuída à presença de macroporos e mesoporos interconectados, ${ }^{25,26}$ o que contribui não só para a penetração de fluidos corpóreos através da estrutura como também para o processo de biodegradação do material. ${ }^{27}$

Os dados da determinação de iodo por AAN nas matrizes de hidroxiapatita estão apresentados na Tabela 2. O teor de iodo é dado em porcentagem $(\mathrm{m} / \mathrm{m})$ da matriz impregnada. A quantidade de iodo impregnada na matriz variou com a concentração da solução de NaI; quanto mais concentrada a solução maior a massa de iodo encontrada nas matrizes, mostrando que elas apresentam uma alta porosidade aberta.

(b)

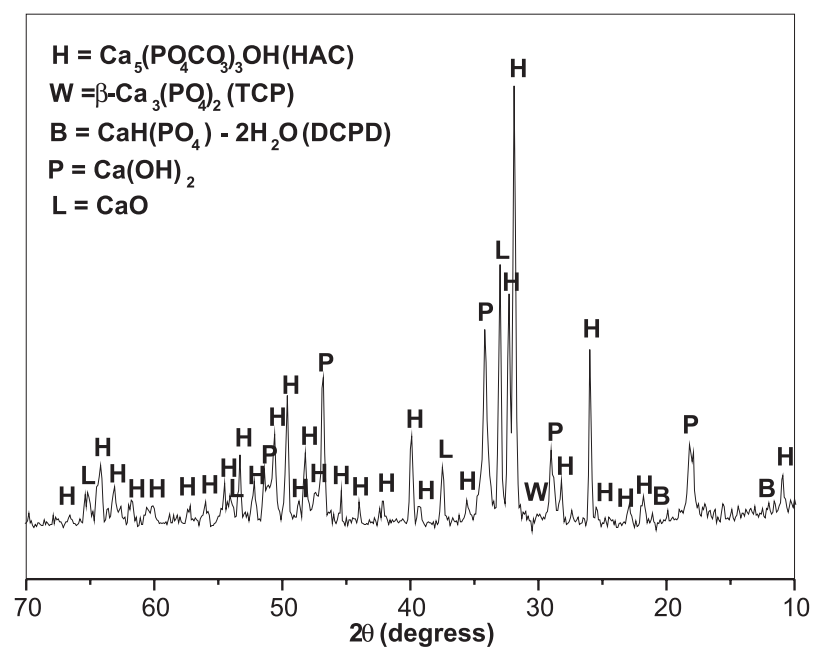

Figura 2. Difratogramas dos pós de HA (a) e HACL (b)

Tabela 1. Dados experimentais dos pós e matrizes de HA e HACL

$$
\text { Dados dos Pós }
$$

Dados das Matrizes

Amostras

Composição Química

\begin{tabular}{|c|c|c|c|c|c|c|c|c|}
\hline & $\mathrm{Ca} \%(\mathrm{~m} / \mathrm{m})$ & $\mathrm{P} \%(\mathrm{~m} / \mathrm{m})$ & $\mathrm{Ca} / \mathrm{P}($ molar $)$ & $\mathrm{A}_{\mathrm{s}}\left(\mathrm{m}^{2} / \mathrm{g}\right)$ & $\rho P I\left(g / m^{3}\right)$ & $\rho G\left(g / m^{3}\right)$ & $\mathrm{PA}(\%)$ & PT $(\%)$ \\
\hline HA & $39,00 \pm 0,90^{(1,2)}$ & $18,70 \pm 1,07^{(1,2)}$ & $1,62 \pm 0,13^{(1)}$ & $17,18 \pm 3,88^{(1)}$ & $1,41 \pm 0,0,5^{(1)}$ & $1,36 \pm 0,01^{(1)}$ & $53,60 \pm 0,20^{(1)}$ & $55,30 \pm 1,35^{(1)}$ \\
\hline HACL & $37,10 \pm 10,08^{(1,2)}$ & $18,40 \pm 0,62^{(1,2)}$ & $1,56 \pm 0,01^{(1)}$ & $31,31 \pm 1,25^{(1)}$ & $1,44 \pm 0,01^{(1)}$ & $1,40 \pm 0,01^{(1)}$ & $52,60 \pm 0,51^{(1)}$ & $54,40 \pm 0,50^{(1)}$ \\
\hline
\end{tabular}

$\mathrm{A}_{\mathrm{S}}$ - Área superficial específica. $\rho \mathrm{PI}$ - Densidade método penetração e imersão. \$G - Densidade geométrica. PA - Porosidade aberta. PT Porosidade total. ${ }^{(1)}$ Nível de confiança $=95 \%$. ${ }^{(2)}$ A determinação de Ca e P para os pós de HACL foi realizada com duas amostras e para HA com quatro amostras. 
(a)

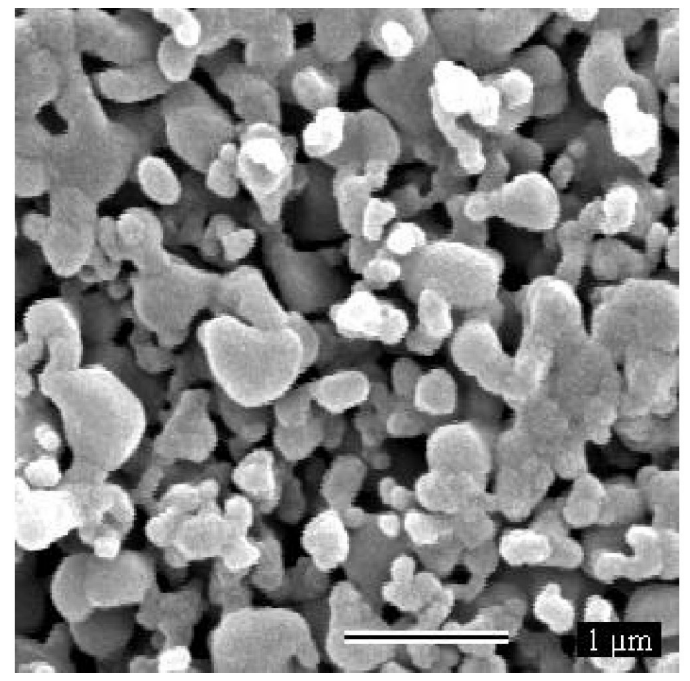

(b)

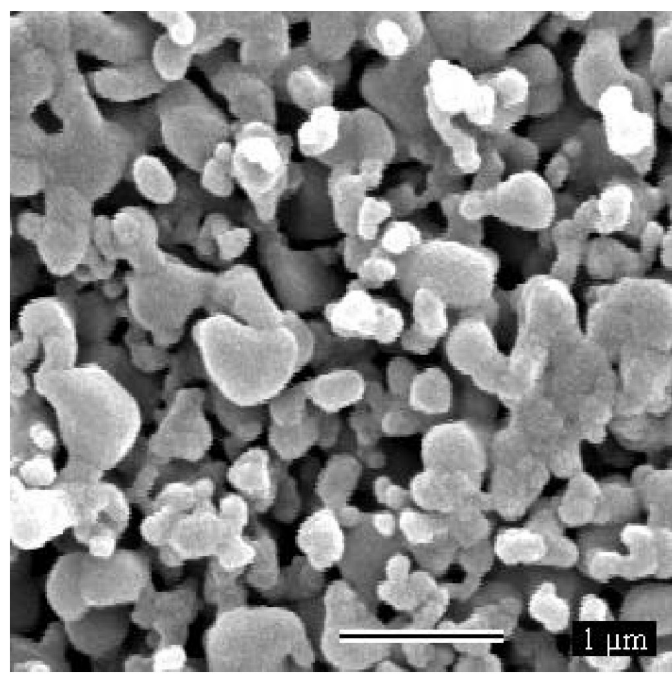

Figura 3. MEV das matrizes sinterizadas de HA tiradas do topo: (a) aumento de 10.000 vezes; (b) aumento de 20.000 vezes

(a)

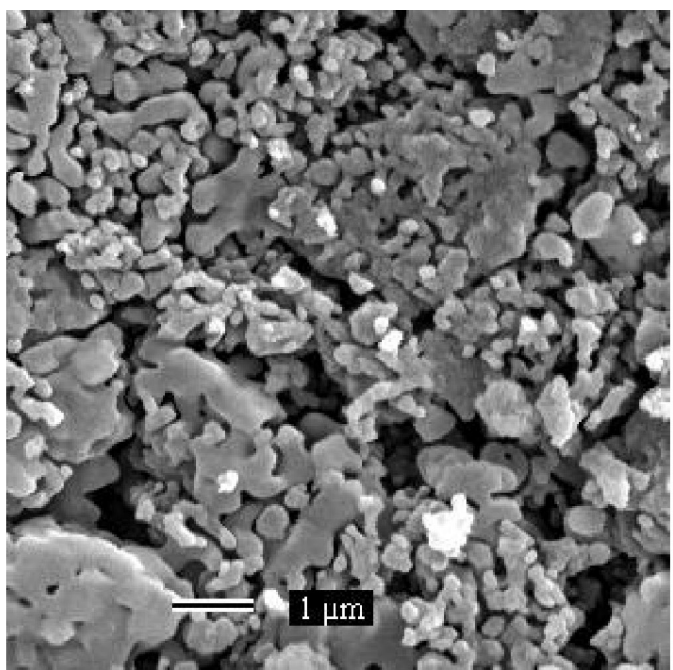

(b)

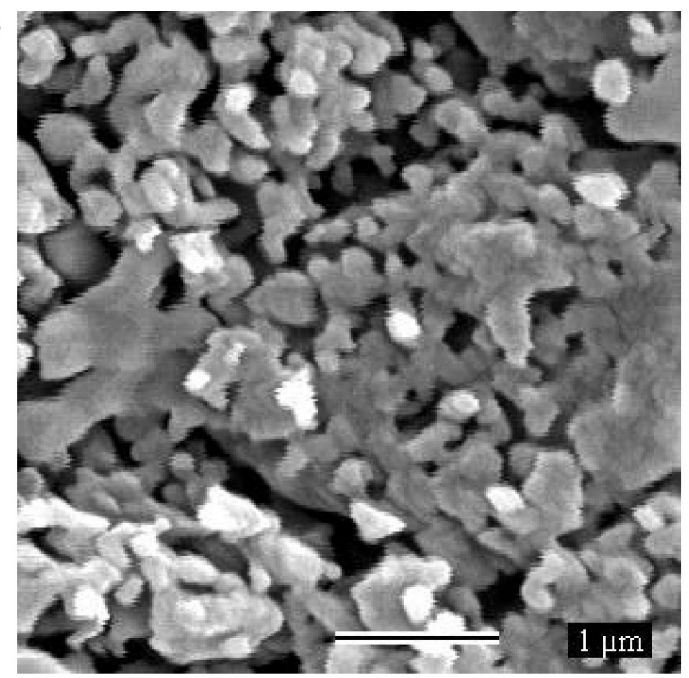

Figura 4. MEV das matrizes sinterizadas de HACL tiradas do topo: (a) aumento de 10.000 vezes; (b) aumento de 20.000 vezes

Uma comparação entre as massas de iodo (determinada pela técnica de AAN e calculada através de estequiometria de reação) foi realizada para validar o processo de impregnação (Tabela 2). A partir dos valores de massa de iodo observou-se que o processo de impregnação é eficiente e depende somente da concentração da solução.

Comparando as massas de iodo medida e calculada mostradas na Tabela 2, nota-se que para as soluções a $1,0 \mathrm{~mol} / \mathrm{L}$ as variações entre a massa de iodo calculada e a medida por AAN são menores que para as soluções de $0,5 \mathrm{~mol} / \mathrm{L}$, revelando uma maior eficiência para os processos de impregnação com soluções mais concentradas. Durante o processo de impregnação, foi utilizado o iodo não radioativo. Espera-se que o iodo-131 (radioativo) tenha o mesmo comportamento na impregnação que o iodo - 127 (isótopo estável). Por isso, foram reproduzidos os mesmos parâmetros de $\mathrm{pH}$ e de temperatura que o fabricante usa para gerar a solução de iodeto de sódio radioativo. ${ }^{22,23} \mathrm{~A}$ obtenção deste rádiofarmaco no mercado não é difícil, os fabricantes disponibilizam uma variedade de produtos com diferentes parâmetros. O parâmetro mais importante é a atividade da solução, por ter relação direta com a dose requerida no procedimento de braquiterapia. Esta variedade de atividades é de grande importância, uma vez que está relacionada à quantidade de solução que pode ser incorporada na matriz para se atingir a dose desejada no procedimento de braquiterapia.
As curvas de degradação para as amostras de matrizes cerâmicas HA e HACL, em termos de liberação de cálcio e de fosfato em função do tempo de imersão no meio, estão apresentadas na Figura 5. Perfis de liberação de cálcio e fosfato similares foram observados para os dois tipos de matrizes avaliadas, exibindo cinéticas muito rápidas até a primeira hora de experimento, com liberação cumulativa de até $0,15 \%$ de cálcio e $0,22 \%$ de fosfato para HA, e $0,12 \%$ de cálcio e $0,057 \%$ de fosfato para HACL. Entretanto, para tempos subsequentes, cinéticas mais lentas ou tendendo a constante (para liberação de fosfato pela amostra HACL) foram observadas com máximos de liberação cumulativa da ordem de $0,28 \%$ de cálcio e $0,45 \%$ de fósforo para HA, e de $0,23 \%$ de cálcio e $0,082 \%$ de fosfato para HACL. A dissolução parcial de cerâmicos de fosfatos de cálcio, como é o caso das amostras de HA e HACL, em meio fisiológico resulta na liberação de íons $\mathrm{Ca}^{2+}, \mathrm{PO}_{4}^{3-}$, $\mathrm{HPO}_{4}^{2-} \mathrm{C}_{3}{ }^{2-}$, e na supersaturação do meio com respeito às fases de cálcio e fósforo. O comportamento degradável desses cerâmicos é função não só das condições do meio fisiológico, mas também da sua composição e tipos de fases agregadas, do seu grau de cristalinidade e de suas características microestruturais. ${ }^{16,28-32}$ Misturas de fases solúveis como o fosfato de tricálcio (TCP) e o fosfato de dicálcio (DCP) bem como apatitas carbonatadas $\left(\mathrm{CO}_{3}\right.$-Ap) estão presentes na composição das amostras de HA e HACL. ${ }^{10,13}$ Essas fases solúveis degradam mais rapidamente que a hidroxiapatita estequiométrica $(\mathrm{Ca} / \mathrm{P}=1,67)$ que é 
Tabela 2. Quantificação de iodo nas matrizes

\begin{tabular}{lccccccc}
\hline Amostra & $\begin{array}{c}\text { Solução NaI } \\
(\mathrm{mol} / \mathrm{L})\end{array}$ & $\begin{array}{c}\text { Massa da matriz } \\
\text { seca }(\mathrm{g})\end{array}$ & $\begin{array}{c}\text { Massa da matriz } \\
\text { impregnada }(\mathrm{g})\end{array}$ & $\begin{array}{c}\text { Teor de I }(\%) \\
\text { AAN }\end{array}$ & $\begin{array}{c}\text { Massa de I } \\
\text { medida }(\mathrm{g}) \text { AAN }\end{array}$ & $\begin{array}{c}\text { Massa de I } \\
\text { calculada }(\mathrm{g})\end{array}$ & $\begin{array}{c}\text { comparação } \\
(\%)\end{array}$ \\
\hline HA & 1,0 & 0,2754 & 0,3748 & $3,5 \pm 0,4$ & 0,0128 & 0,0131 & 99,1 \\
HA & 0,5 & 0,2807 & 0,3861 & $2,1 \pm 0,2$ & 0,00788 & 0,00819 & 69,1 \\
HACL & 1,0 & 0,2758 & 0,3756 & $3,4 \pm 0,3$ & 0,0125 & 0,0128 & 98,9 \\
HACL & 0,5 & 0,2810 & 0,3799 & $2,1 \pm 0,2$ & 0,00790 & 0,00798 & 72,4 \\
\hline
\end{tabular}

relativamente estável em condições in vitro and in vivo. ${ }^{16}$ As curvas de solubilidade para os diferentes tipos de fosfatos de cálcio revelam que o grau de dissolução é função do tipo e da quantidade de fases solúveis presentes na composição do cerâmico, ${ }^{30-32}$ e que cerâmicos de fosfatos de cálcio com razão molar $\mathrm{Ca} / \mathrm{P}$ compreendida na faixa de 1 e 2 estão sujeitos à biodegradação. Adicionalmente, a formação de microcristais com razão molar $\mathrm{Ca} / \mathrm{P}$ similar àquelas correspondentes aos cristais de apatitas deficientes em cálcio $(\mathrm{Ca} / \mathrm{P} \neq 1,67)$ está diretamente relacionada à razão inicial $\beta$-TCP/hidroxiapatita em cerâmicos de fosfatos de cálcio bifásicos. Quanto maior for a razão $\beta$-TCP/hidroxiapatita, mais favorecida será a precipitação de cristais de hidroxiapatita deficiente em cálcio ou carbonatada nos microporos do cerâmico e, dessa forma, é possível controlar a cinética de dissolução e precipitação. Com base nessa análise, a menor dissolução de fósforo (em termos de fosfato) observada para a amostra de HACL $(\mathrm{Ca} / \mathrm{P}=1,56)$ em comparação à $\mathrm{HA}(\mathrm{Ca} / \mathrm{P}=1,56)$ pode estar associada à precipitação de cristais de apatita carbonatada nos poros da amostra, favorecendo uma menor dissolução (liberação) de fosfatos no meio (Figura 4). O comportamento cinético exibido pela curva de liberação de cálcio e de fosfato sugere que o mecanismo de degradação das matrizes de HA e HACL em soro fisiológico, sob as condições investigadas, é governado por processo de difusão fundamentado na $1^{\text {a }}$ lei de difusão de Fick, segundo modelo matemático de Higuchi (T. Higuchi, T.; J. Pharm. Sci. 1963, 52, 1145). Esse modelo de difusão supõe que uma matriz porosa degradável em uma fase líquida se dissolve lentamente no meio a partir da penetração do líquido através de seus poros. O material dissolve-se lentamente na fase líquida e difunde através da estrutura da matriz pelas interconectividades entre os poros. Matematicamente, a Equação de Higuchi (4) pode ser usada para calcular as taxas de liberação de cálcio e fosfato no soro e ser representada como:

$\mathrm{M}=\mathrm{kt}^{1 / 2}$

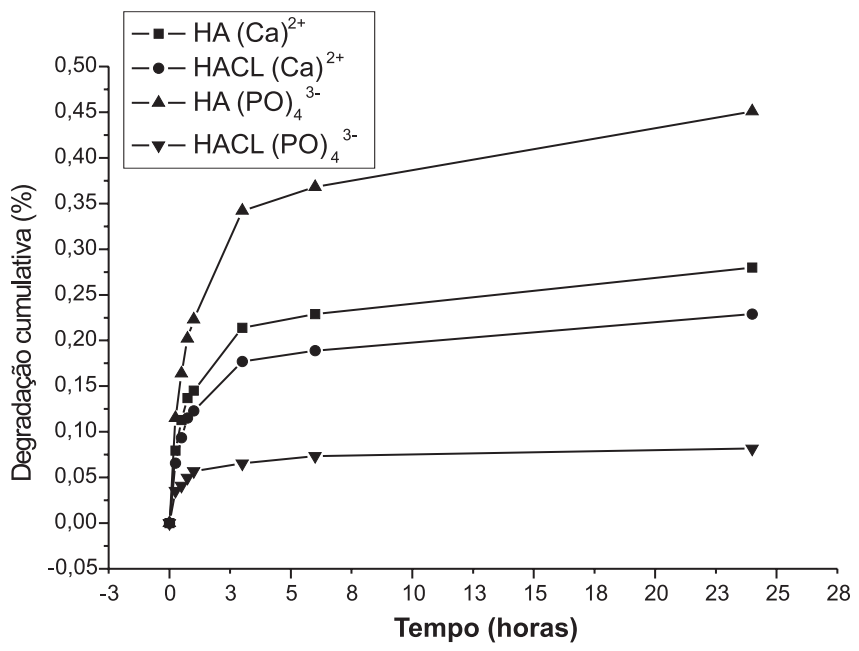

Figura 5 . Curvas de degradação das matrizes cerâmicas HA e HACL, em termos de liberação de cálcio $\left(\mathrm{Ca}^{2+}\right)$ e fosfato $\left(\mathrm{PO}_{4}^{3-}\right)$ onde $\mathrm{M}$ é a quantidade total de material liberado no tempo t, e k é a constante cinética de liberação que leva em conta as características estruturais e geométricas da amostra.

Correlações entre a quantidade percentual cumulativa de cálcio ou fosfato liberado no meio em função da raiz quadrada do tempo foram avaliadas usando-se regressão linear incluindo a origem entre os dados, com coeficientes de correlação exibindo desvios de linearidade não significativos $(r=0,99)$. Na análise foram consideradas duas cinéticas, a primeira levando-se em conta os dados de liberação até a primeira hora de experimento e a segunda, os dados relativos aos tempos subsequentes. Duas equações lineares foram obtidas, indicando que as liberações de cálcio e fosfato são reguladas por dois mecanismos de difusão. Valores de taxa de liberação para cálcio e fosfato, expressos em termos das respectivas constantes cinéticas k, e os coeficientes de

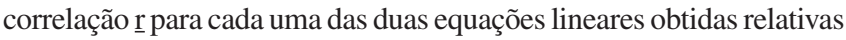
às amostras de HA e HACL estão apresentados na Tabela 3.

Não foram observadas diferenças significativas entre as constantes cinéticas de liberação de cálcio para ambas as amostras investigadas. Por outro lado, valores de constantes cinéticas significativamente maiores para liberação de fosfato foram encontrados para a amostra HA em relação à HACL. Como discutido anteriormente, esse fato pode estar associado à possível presença de fases mais solúveis, como TCP e DCP, na composição da matriz $\mathrm{HA}^{10,13}$ que favorecem uma maior dissolução da matriz no meio, ${ }^{16,30,32}$ embora ela apresente um valor de razão molar $\mathrm{Ca} / \mathrm{P}(1,62)$ mais próximo ao estequiométrico $(1,67)$ e não contenha quantidades significativas de carbonato em sua composição diferentemente da amostra HACL. ${ }^{10,13}$

Tabela 3. Parâmetros cinéticos de liberação de cálcio e fosfato para as amostras HA e HACL

\begin{tabular}{lcccc}
\hline Amostra & $\begin{array}{c}\mathrm{K}_{\text {cálcio }} \\
\left(\% . \mathrm{h}^{-1 / 2}\right)\end{array}$ & $\begin{array}{c}\mathrm{K}_{\text {fosfato }} \\
\left(\% . \mathrm{h}^{-1 / 2}\right)\end{array}$ & $\mathrm{r}_{\text {cálcio }}$ & $\mathrm{r}_{\text {fosfato }}$ \\
\hline HA & 0,15 & 0,23 & 0,99 & 0,99 \\
HACL & 0,021 & 0,034 & 0,99 & 0,99 \\
& 0,13 & 0,056 & 0,99 & 0,99 \\
& 0,016 & 0,0059 & 0,99 & 0,99 \\
\hline
\end{tabular}

\section{CONCLUSÃO}

As matrizes de HA e HACL apresentaram características de hidroxiapatita bioabsorvível e presença de macro e mesoporos interconectados com densidade média da ordem de $45 \%$ da densidade teórica, mostrando serem altamente porosas, com porosidade total da ordem de $55 \%$ e porosidade aberta da ordem de $54 \%$.

Utilizando a solução aquosa de iodeto de sódio foi possível incorporar iodo na porosidade aberta através de um processo de impregnação, possibilitando a obtenção de matrizes com diferentes quantidades de iodo incorporado (variando a concentração da solução de $\mathrm{NaI}$ ), podendo assim variar as atividades das fontes radioativas.

A construção de fontes para braquiterapia bioabsorvível utilizando hidroxiapatita é potencializada devido à flexibilidade apresentada neste 
trabalho, pois as matrizes apresentaram uma faixa de atividade ampla, entre baixas e altas atividades, facilitando o uso do material.

O modelo de Higuchi, fundamentado na $1^{a}$ lei de difusão de Fick, foi sugerido para explicar os perfis cinéticos de liberação de cálcio e fosfato para as amostras investigadas. Diferenças significativas observadas entre os valores de taxas de liberação de fosfato para os dois tipos de amostras avaliadas podem ser explicadas em termos da presença de misturas de fases solúveis, como TCP e DCP bem como $\mathrm{CO}_{3}$-Ap, na composição das amostras investigadas, responsáveis pelo controle de mecanismos cinéticos de dissolução e precipitação.

\section{AGRADECIMENTOS}

Ao apoio financeiro do CNPq e do CDTN/CNEN, à colaboração do Laboratório Materiais e Combustível Nuclear do Serviço de Materiais e Combustível Nuclear e ao Serviço de Reator e Irradiações, pela realização das análises por ativação nêutronica, ambos do Centro de Desenvolvimento da Tecnologia Nuclear, Laboratório de Microscopia Eletrônica e Microanálise do Departamento de Física/ UFMG do consórcio UFMG-CDTN.

\section{REFERÊNCIAS}

1. Hsu, C. A.; Termochimica 2002, 392-393, 157.

2. Ivanova, T. I.; Frank-Kamenetskaya, O. V.; Kol'tsov, A. B.; Ugolkov, V. L.; J. Solid State Chem. 2001, 160, 340.

3. Kinari, A. B.; Gross, K. A.; Biomaterials 2004, 25, 4935.

4. Milev, A.; Kannangara, G. S. K.; Bem-Nissan, B.; Mater. Lett. 2003, 57, 1960.

5. Silva, V. V.; Lameiras, F. S.; Domingues, R. Z.; Composites Science and Technology 2001, 61, 301

6. Itokazu, M.; Yang, W.; Aoki, T.; Ohara, A.; Kato, N.; Biomaterials 1998, 19, 817 .

7. Coniglione, R.; Russel, J. L. Jr.; IBT Technology Partners, WO 97/19706 1997.

8. Nath, R.; Anderson, L. L.; Olch, A. J.; Stitt, J. A.; Williamson, J. F.; Med. Phys. 1997, 24, 1567.

9. Leavitt, R. D.; Avila, L.; Focal, Inc. WO 97/33628 1997.

10. Lacerda, K. A.; Lameiras, F. S.; Silva, V. V.; Revista Escola Minas 2006, $59,217$.
11. Lacerda, K. A.; Lameiras, F. S.; Silva, V. V.; Brazilian Archives of Biology and Technology 2007, 50, 707.

12. Chen, T. M.; Wang, H. J.; Chen, S. L.; Lin, F. H.; Ann. Plast. Surg. 2004, $52,303$.

13. Lacerda, K. A.; Dissertação de Mestrado, Centro de Desenvolvimento da Tecnologia Nuclear, Comissão Nacional de Energia Nuclear, Brasil, 2005.

14. Silva, V. V.; Tese de Doutorado, Universidade Federal de Minas Gerais, Brasil, 1998.

15. Oonishi, H.; Oomamiuda, K. Em Handbook of Biomaterials Properties; Black, J.; Hastings, G., eds.; Chapman \& Hall: London, 1998.

16. Legreros, R. Z.; Clinical Materials 1993, 14, 65.

17. Narasaju, T. S. B.; Pheba, D. E.; J. Mater. Sci. 1996, 31, 1.

18. Silva, V. V.; Fernandes, R. Z. D.; Cerâmica 1996, 42, 276.

19. Ferreira, R. A. N.; Tese de Doutorado, Universidade Estadual de Campinas, Brasil, 2000

20. Faghihian, H.; Ghannadi-Maragheh, M.; Malekpour, A.; J. Radioanal. Nucl. Chem. 2002, 254, 545.

21. Santos, A. M. M.; Vasconcelos, W. L.; J. Non-Cryst. Solids 2000, 273, 145.

22. Amersham Biosciences Limited; Biodirectory 2002, 130, 138.

23. http://www.ipen.br/conteudo/upload/200808150747480.produtos_ servicos_13082008.pdf, acessada em Maio 2009.

24. Menezes, M. A. B. C.; Tese de Doutorado, Universidade Federal de Minas Gerais, Brasil, 2002.

25. Murugam, R.; Ramakrishna, S.; Biomaterials 2004, 25, 3829.

26. Fabri, M.; Celotti, G. C.; Ravaglioli, A.; Biomaterials 1995, 16, 225.

27. Landi, E.; Celotti, G.; Logroscino, G.; Tampieri, A.; J. Eur. Ceram. Soc. 2003, 23, 2931.

28. Legreros, R. Z. Em Monographs in Oral Sciences; Myers, H. M., ed.; Karger: Basel, 1991, vol. 15, p. 4-45.

29 Legreros, R. Z.; LeGeros, J. P.; Daculsi, G.; Kijkowska, R. Em Encyclopedic handbook of biomaterials and bioengineering, Part A Feng Huei, 2; Wise, D. I., ed.; Marcel Dekker: New York, 1995, p. 1429-1463.

30. Lin, F. H.; Liao, C. J.; Chen, K. S.; Sun, J. S.; Liu, H. C.; Biomaterials 1997, 18, 915

31. Reser, M. K.; Am. Ceram. Soc. 1964, 246.

32. Groot, K. Em Biocompatibility of clinical implant materials; Williams, D. F., ed.; CRC Press: Boca Raton, 1984, p. 199-222. 